\title{
Freudian unconscious and cognitive neuroscience
}

Freudian Unconscious and Cognitive Neuroscience. By Vesa Talvitie. London: Karnac Books Ltd, 2009.

\begin{abstract}
... [T]he psychological unconscious documented by latter-day scientific psychology is quite different from what Sigmund Freud and his psychoanalytic colleagues had in mind in fin de siècle Vienna. Their unconscious was hot and wet; it seethed with lust and anger; it was hallucinatory, primitive, and irrational. The unconscious of contemporary psychology is kinder and gentler than that and more reality bound and rational, even if it is entirely cold and dry.
\end{abstract}

Kihlstrom, Barnhardt and Tataryn (p. 789),' quoted in Talvitie (p. 2)²

It was this 'hot and wet' perception of Freud's notion of the unconscious that first attracted me to psychoanalysis. However, the question regarding whether, when neuroscientists speak of unconscious processing, they are speaking in the same terms as psychoanalysts or not, has become of increasing interest to me. It was, therefore, my sense that Talvitie's work served as something of a bridge between the two paradigms that drew me to it.

As is the case with all mental phenomena, the questions about just how and where the unconscious might be are associated with the mind-body problem that distinguishes monists and dualists. For monists, mind emerges from brain activity. Dualists hold that mind and brain are separate and cannot be reduced to each other. Dualism has largely fallen out of favour with most neuroscientists, and Talvitie 2 himself is clearly a monist. At the same time, while there is consensus that mind or consciousness emerges from neurophysiological processes, there remains a lack of clarity as to how this is achieved. Talvitie (p. 50) ${ }^{2}$ goes as far as to make the point that cognitive science has little doubt that unconscious matters determine our behaviour. Rather, it is the role and emergence of consciousness that is in question.

At the same time, the two are clearly related. It would seem that consciousness is a limited product of the functioning of the mind. We know we can hold only seven units of information in working memory, and most people are able to retain only four units of visuospatial information. What happens to other information? Is there other information? If there is, is it processed unconsciously?

These questions have been asked for a long time now, and various routes have been utilised by neuroscientists in their quest to answer them. Benjamin Libet (Talvitie, ${ }^{2}$ p. 50) has shown that readiness potential occurs 220 - 550 milliseconds before the conscious decision to move one's finger. In addition to subliminal priming, split-brain studies have been used to show that subjects presented with sensory input to one hemisphere do not perceive this input from the other hemisphere. Another route has related to analyses of memory processes. We know that explicit or declarative memory, which is the memory for facts and events, depends on the hippocampus and is stored in a distributed network throughout the brain. Implicit or procedural memory, on the other hand, is the learning of various skills and relies on changes to the domain-specific neural processors involved in that skill, so that, for example, motor regions will be involved in the learning of a finger sequencing task. Amnesics exhibiting a deficit in explicit memory continue to retain their ability to learn new skills and habits, so that although they may not recall the learning event, their performance is influenced by this experience.

The psychoanalytic idea that a memory may be stored in the unconscious, only to emerge intact at a later stage, has however been challenged by neuroscience. Cognitive neuroscience has revealed that our memory processes are highly subject to error. As we grow older, source memory or our memory of the context in which an event took place begins to fail. As Martha Weinman Lear (p. 106) explains, various emotions such as guilt or desire, associated with a particular memory, may affect our recollection of it. 'We are always dealing with (A) the event as it actually happened, overlaid by (B) how we think it might have happened, further modified by (C) how we believe that it should have happened, and additionally burdened by (D) how we wish that it had happened or (E) how we fear that it actually happened.' Furthermore, the accretive nature of memory formation ensures that other factors such as language may play tricks on our recollections. For example, it has been shown that the act of repeating a false statement over and over leads people to believe increasingly in its veracity. ${ }^{4}$

Elizabeth Loftus ${ }^{5}$ has produced an impressive amount of research showing how easy it is to create false memories of past events. In one study, participants watched a film of a car accident. Researchers asked some subjects how fast the cars were going when they 'smashed into' each other and others how fast the cars were going when they 'hit' each other. The subjects who heard the word 'smashed' gave significantly higher estimates of the speed than those who heard the word 'hit'. In another experiment, subjects were fed incorrect information about an accident after watching a film of it. For example, they were asked whether a traffic light had turned yellow before the collision, when in fact the light was green. This resulted in many then remembering a yellow light that had never existed (p. 868). ${ }^{5}$

With regard to memory consolidation, however, matters are rather more complex. On the one hand, it appears that stress-related hormones and the activation of the amygdala will produce strong memories in animal and human subjects who are victims of isolated acts of trauma, so that such emotion-laden memories tend not to be forgotten. If a person rapes or attacks you, inspiring strong emotion in you, the chances are good that you will remember the experience and not repress it. At the same time, as happens with people who are repeatedly under extreme stress, such as soldiers in heavy war zones or victims of extended sexual abuse, excessive exposure to these very same stress hormones damages neurons and thus leads to traumainspired memory impairment. ${ }^{6}$

Talvitie $^{2}$ garners data regarding a cognitive neuroscientific understanding of unconscious processes and structures it according to a four-level model, the starting point of which is the cognitivist 
and monistic idea that conscious states are always the end-products of several neural and conscious processes. His first level is, therefore, that of neurophysiological processes, which lead to the second level, that of consciousness (p. 93). ${ }^{2}$ While, according to Talvitie (p. 94), the Freudian view concerning consciousness and the unconscious tends to be digital, in that ideas and mental states are either conscious or unconscious (the term preconscious does not change the picture very much), his four-level model delineates consciousness and unconsciousness as a continuum. Hence, we are not either conscious or unconscious on matters, but rather less or more conscious.

The brain with its orchestration of neural activity gives rise to ideas, feelings and associations. As this happens, it makes a considerable difference to awareness whether an idea or feeling takes part in the stream for 200 milliseconds, for a minute, or for hours or days (p. 24). ${ }^{2}$ So, when an idea or feeling appears and disappears rapidly in the scope of consciousness, it is probably not remembered later. From the perspective of one's self-understanding or self-narrative, it makes no difference whether an idea has never appeared in consciousness, or if it appeared there for one fleeting moment.

The mechanism that picks up particles from the stream of consciousness for closer examination is attention. Attention may also be directed to one's bodily reactions, and affairs taking place in the surrounding world, bringing us to the third level of the model, namely self-consciousness. In Talvitie's view (p. 96), a fish is conscious of another fish swimming in front of it and an infant is conscious of the light being switched off. Both fish and infants possess conscious states, but they are not self-conscious because they do not possess ideas regarding their personal characteristics, i.e. their sex, age, unique life history, idiosyncratic ways of reacting to stimuli, and so forth.

Essential to an understanding of self-consciousness are two terms. The first, 'phenomenal consciousness', refers to the particular content and quality of a state of consciousness, i.e. whatever pains, pleasures and other feelings might be associated with it.

The second term is 'access-consciousness'. This refers to the extent to which we are aware of a particular state. For example, when we are in one conscious state, we might or might not be aware of other states. Restriction of access to consciousness is a common phenomenon. When concentrating on writing, we may not be aware of feelings of hunger; a soccer player trying to score a goal might not notice an otherwise painful leg injury. Many competencies are indeed facilitated by restricting access to other competing sensations in consciousness, making us unaware of them in our perception of the flow of our experiences. We are only able to attach meaning and significance to a particular state if it is accessible to us.

In the domain of psychoanalysis, the restriction of access between conscious states is called dissociation, and it is often used to refer to cases involving extreme psychopathology. However, Talvitie ${ }^{2}$ notes that a degree of dissociation is a part of normal cognitive functioning. He cites empirical research supporting the idea that repression too is a form of dissociation, in that it too is context-dependent. ${ }^{6}$ Hence, what might be repressed in one situation may become conscious in another.

Similarly to Freud, Talvitie ${ }^{2}$ appears to view defences as referring to a difficulty with assimilating emotionally valent information. So, should we hear tragic news, we may respond inappropriately by staying calm, laughing hysterically or denying it, until such time as we are able to process the emotional significance of the facts we are presented with and so integrate them within our personal narrative, memories, feelings and self-consciousness.

This may be a good explanation of denial, but what about the repression of unacceptable impulses that also form a part of Freud's model of the mind, the inhibitory capacity that gave Freud's ego executive control over the otherwise automatic, biologically determined functions of the mind? Talvitie ${ }^{2}$ does not pay sufficient attention to this type of defence, and it becomes necessary to fill the vacuum with the work of Solms and Turnbull, who show how repression is intimately linked to the functioning of the prefrontal lobes. These parts of the brain integrate information streaming into the brain from the body and its environment with information derived from previous experiences stored elsewhere in the brain. They then act to calculate the best course of action before executing a motor response. Solms and Turnbull (p. 287) ${ }^{8}$ argue that 'repression' is therefore consonant with a short-circuiting of this process, so that repressed information is information that is excluded from the overarching network of executive control exercised by the prefrontal lobes (p. 287)? Patients who reveal ego deficits, who are unable to identify contradictory beliefs and whose perception of reality is submerged in their wishful fantasies have suffered bilateral deep frontal lobe lesions, as noted by Solms and Turnbull (p. 260). ${ }^{8}$

However, this is not the whole story; not only do the lesions noted above affect the individual's capacity to modulate his or her behaviour, they also affect his or her inner speech, the speech or overt thought with which we regulate our behaviour in terms of verbal programmes, such as 'first I must do this, then when it is finished, do that'. This 'inner speech' fits into Damasio's ${ }^{9}$ autobiographical self and Talvitie's (p. 98) fourth level, which is that of narrative self-consciousness. The fish and the infant, described earlier with regard to the third level, are unable to identify what their current feelings are compared with those they had yesterday; and they do not understand that life is finite. These ideas are generally coded and stored by means of language. Language also gives rise to abstract thinking, so that we become able to create fictional worlds and to work with ideas. Language helps to make it possible for us to understand ourselves and others as possessing certain mental characteristics and a unique personal history.

Although Talvitie's model emerges largely from the cognitive tradition, it also shares Bion's focus on language as a means through which poorly elaborated experiences and sensations are transformed into thoughts than can be entertained and tolerated, despite the discomfort that may accompany them (see Ivey ${ }^{10}$ ). Talvitie (p. 93) ${ }^{2}$ also 
links it to Peter Fonagy and Mary Target's work on mentalisation and describes it as a possible elaboration and extension of this work.

However, drawing on Habermas and Wittgenstein, Talvitie reminds us (p. 99) that each language community has its own vocabulary for mental matters, and we have all been socialised to adopt the folk psychology of the surrounding culture. Language functions less to represent the state of things objectively, and more to make things happen in the social world. Consequently, our verbal expressions of our mental states are not true or false at first hand. Instead, they are more or less realistic or appropriate given the data, and the conventions prevailing in one's language community. Language is a cultural phenomenon, and cultural conventions provide the frames, and also set the limits with which we represent our phenomenal reality (p. 100). ${ }^{2}$

Our cognitions and narratives regarding ourselves may also be biased, as humans have a tendency to see and narrate themselves in one way, and a resistance to seeing and narrating themselves in other ways (p. 101). ${ }^{2}$ Perhaps this is because, as Dennett (p. 418) ${ }^{10}$ puts it, the self has to be controlled, protected and defined, and this occurs through our telling stories of what we are like. The boundaries of the self also change, so that sometimes we enlarge our boundaries and at other times, in response to perceived challenges real or imaginary, we let our boundaries shrink (p. 417)."1 Translated into the language of psychoanalysis, we can say that we have an ideal self or self-image that might become threatened, giving rise to resistance and the activation of defences (p. 102).

So, for example, to go back to the model, Talvitie ${ }^{2}$ explains how one might understand Freud's identification of repressed oedipal wishes as follows. A person has conflictual feelings in relation to his or her parents. On the one hand, there are fleeting sexual and aggressive feelings that the person in question does not attend to, and so they become less accessible or remembered. At the same time, the person also has fantasies and fears regarding how a parent of the same sex might suffer an accident. In analysis, with the request that the analysand mention everything that comes to his or her mind, attention becomes directed on those sexual and aggressive feelings that have hitherto been fleeting and unattended to. The analyst or analysand then draws together various feelings, fears and fantasies, by making use of an anticipated oedipal logic of association in order to claim the existence of repressed oedipal ideas (p. 98). ${ }^{2}$

Unfortunately Talvitie ${ }^{2}$ does not sufficiently develop the implications of language as playing a role in framing our experience of the world and thus functioning as a conceptual system that determines to some extent what we choose to attend to or not. His ideas are, nevertheless, clearly founded on Freud's ${ }^{12}$ early conceptualisation of the unconscious, where he argued that attaching words to thoughts makes it possible to bring those thoughts to consciousness, and of Fromm's ${ }^{13}$ understanding of what he termed the social unconscious.

Fromm ${ }^{13}$ acknowledges that pain, sexual desire and hunger, as sensations that are relevant to survival, have easy access to awareness.
However, '[w]hether or not subtle effective experiences can arrive at awareness depends on the degree to which such experiences are cultivated in a given culture' ( $p$ 109)..$^{13}$ Drawing on Fromm, ${ }^{13}$ one might then think of an example where a family espouses an ideology that teaches that everything that father does is always right. Father's actions are then prefaced in these terms, so that a child of the family also believes initially that everything the father does is always right. If the father punishes the child unfairly, he or she will not be able to understand the father's actions as cruel or misguided and if, for an instant, the child doubts the wisdom of the father's actions, it is possible that he or she will not pay attention to this doubt and it will exit awareness. This will be especially true if much of father's behaviour is benevolent and well-considered.

Talvitie ${ }^{2}$ does not explore how conceptual systems filter the social world so that certain experiences enter awareness while others are turned away, and we are forced to turn to Fromm ${ }^{13}$ for an answer in this regard. He identifies language and the prevalent logic directing the thinking of a culture as significant filters. The most important filtering comes about through social taboos which declare certain ideas and feelings to be forbidden, dangerous or improper (p. 114). ${ }^{2}$ Whether social taboos act uniformly on all sectors of society is questionable, however. In Nazi Germany, most Germans surely found it easier to repress information regarding the injustices committed against Jewish people than the latter themselves?

In conclusion, while Talvitie's ${ }^{2}$ account contains many gaps that one needs to fill by looking elsewhere, for example in Solms and Turnbull's ${ }^{8}$ more comprehensive introduction to neuropsychoanalysis, it is nevertheless a thought-provoking work that provides a structure helpful to those interested in identifying bridges between psychoanalytic thinking and cognitive and neuroscientific approaches to our understanding of the mind.

\section{Ethelwyn Eleonore Rebelo}

Department of Psychiatry

University of the Witwatersand

\section{References}

1. Talvitie V. Freudian Unconscious and Cognitive Neuroscience: From Unconscious Fantasies to Neural Algorithms. London: Karnac, 2009.

2. Weinman LM. Where did I Leave My Glasses: The What, When and Why of Normal Memory Loss. London: JR Books, 2008.

3. Kihlstrom JF, Barnhardt TM, Tataryn DJ. The psychological unconscious: found, lost, and regained. Am Psychol 1992;47:788-791.

4. Schacter DL. Searching for Memory: The Brain, the Mind, and the Past. New York: Basic Books, 1996.

5. Loftus EF. Make-believe memories. Am Psychol 2003;8(11):867-873.

6. Rofé Y. Does repression exist? Memory, pathogenic, unconscious and clinical evidence. Rev Gen Psychol 2008;12:63-81.

7. Freud S. The Ego and the Id. Standard Edition, 1923:19:3.

8. Solms M, Turnbull O. The Brain and the Inner World. New York: Other Press, 2002.

9. Damasio A. The Feeling of What Happens. London: Heinemann, 1999.

10. Ivey G, Bion W: Thinking, feeling and the search for truth. In: Watts J, Cockroft K, Duncan N, eds. Developmental Psychology. Cape Town: UCT Press, 2009:1 12-137.

11. Dennett D. Consciousness Explained. Boston, MA: Little, Brown, 1991.

12. Freud S. The Unconscious. Standard Edition, 1915:14:161.

13. Fromm E. Beyond the Chains of Illusion: My Encounter with Marx \& Freud. London: Abacus, 1986. 